\title{
Optical lithography for nanotechnology
}

\section{Donis Flagello, Bill Arnold}

Donis G. Flagello, Bill Arnold, "Optical lithography for nanotechnology," Proc. SPIE 6327, Nanoengineering: Fabrication, Properties, Optics, and Devices III, 63270D (31 August 2006); doi: 10.1117/12.683305

SPIE. Event: SPIE Optics + Photonics, 2006, San Diego, California, United States 


\title{
OPTICAL LITHOGRAPHY FOR NANOTECHNOLOGY
}

\author{
Donis G. Flagello and Bill Arnold \\ ASML US, Inc., 8555 S. River Parkway, Tempe, Arizona, 85284
}

\begin{abstract}
Optical lithography is continually evolving to meet the ever demanding requirements of the micro - and nanotechnology communities. Since the optical exposure systems used in lithography are some of the most advanced and complex optical instruments ever built, they involve ever more complex illuminator designs, nearly aberration free lenses, and hyper numerical apertures approaching unity and beyond. Fortunately, the lithography community has risen to the challenge by devising many inventive optical systems and various methods to use and optimize exposure systems. The recent advancement of water immersion technology into lithography for $193 \mathrm{~nm}$ wavelengths has allowed the numerical aperture (NA) of lithographic lenses to exceed 1.0 or a hyper-NA region. This allows resolution limits to extend to the $45 \mathrm{~nm}$ node and beyond with NA $>1.3$. At these extreme NAs, the imaging within the photoresist is accomplished by not only using water immersion but also using polarized light lithography.

This paper will review the current state-of-the-art in immersion, hyper-NA lithography. We show the latest results and discuss the various phenomena that may arise using these systems. Furthermore, we show some of the advanced image optimization techniques that allow lithographic printing at the physical limits of resolution. In addition, we show that the future of optical lithography is likely to go well beyond the $30 \mathrm{~nm}$ regime using advancements in $193 \mathrm{~nm}$ doublepatterning technology and/or the use of extreme ultra-violet (EUV) optical systems.
\end{abstract}

Keywords: High NA, polarization, imaging, immersion, 193nm

\section{INTRODUCTION}

An optical step and scan system is a highly complex opto-mechanical tool which combines the finest resolution optical lenses and illumination systems with the highest precision mechanical scanning stages and metrology systems. The end-goal is to provide a high productivity, profitable means for making microcircuits on silicon wafers. By using higher and higher numerical apertures (NA), lower wavelengths, and resolution enhancement techniques (RETs), we have been able to push the limits of the optics well beyond would have been expected just a few years ago. The result of this relentless technical advancement is that optical lithography continues to be the engine that drives Moore's Law. Figure 1 shows the resolution needed by various world-wide chip manufacturers as a function of time. The population is divided into Logic, DRAM, and Flash makers. Traditionally, DRAM has been the driver for resolution; however, in recent years, the extensive use of Flash memory has driven this technology to surpass DRAM such that the projected resolution for high volume production will be $\sim 30 \mathrm{~nm}$ in 2011.

Recent extensions of 193nm lithography have used resolution enhancement technology (RET) and hyper-NA immersion optics to obtain resolutions less than $45 \mathrm{~nm}$ half pitch. This has enabled the production of higher density DRAM and flash memories, as well as new generations of multi-core processors. The chief technology improvements have come due to the development of water immersion optics with $\mathrm{NA}>1$, the use of polarized illumination, and the maturation of several low $\mathrm{k}_{1}$ technologies such as model-based optical proximity correction, source-mask optimization, and various forms of double exposure lithography.

To help in the understanding of how this plethora of technology can achieve such resolution improvement, it is worthwhile to examine the fundamental equation that relates lithography and optics to resolution. Typically, the industry has used a modification of the Rayleigh criterion to define the industry progress of diminishing linewidth by change of

Nanoengineering: Fabrication, Properties, Optics, and Devices III

edited by Elizabeth A. Dobisz, Louay A. Eldada, Proc. of SPIE Vol. 6327

63270D, (2006) - 0277-786X/06/\$15 - doi: 10.1117/12.683305

Proc. of SPIE Vol. 6327 63270D-1 
optical parameters. In its most general form, for lines and spaces with a 1:1 ratio, the linewidth (or resolution) is given as,

$$
\mathrm{LW}=k_{1} \frac{\lambda}{n \sin \theta}=k_{1} \frac{\lambda}{N A},
$$

where $\lambda$. is the wavelength and $\theta$ is the limiting angle of the lens, as defined by the marginal ray in a medium (immersion) with an the index of refraction, $\mathrm{n}, N A$ is the numerical aperture, and $\mathrm{k}_{1}$ is related to the difficulty of the process and has a lower limit of 0.25 with partial coherent imaging using a single exposure. This simple equation with 3 parameters governs optical lithography. We note that the complimentary equation for depth of focus (DOF) is given by,

$$
D O F=\frac{\lambda}{n(1-\cos \theta)}=\frac{\lambda}{n\left(1-\sqrt{1-\frac{N A^{2}}{n^{2}}}\right)},
$$

which will result in an increase in DOF, when comparing immersion systems to dry systems assuming a constant NA.

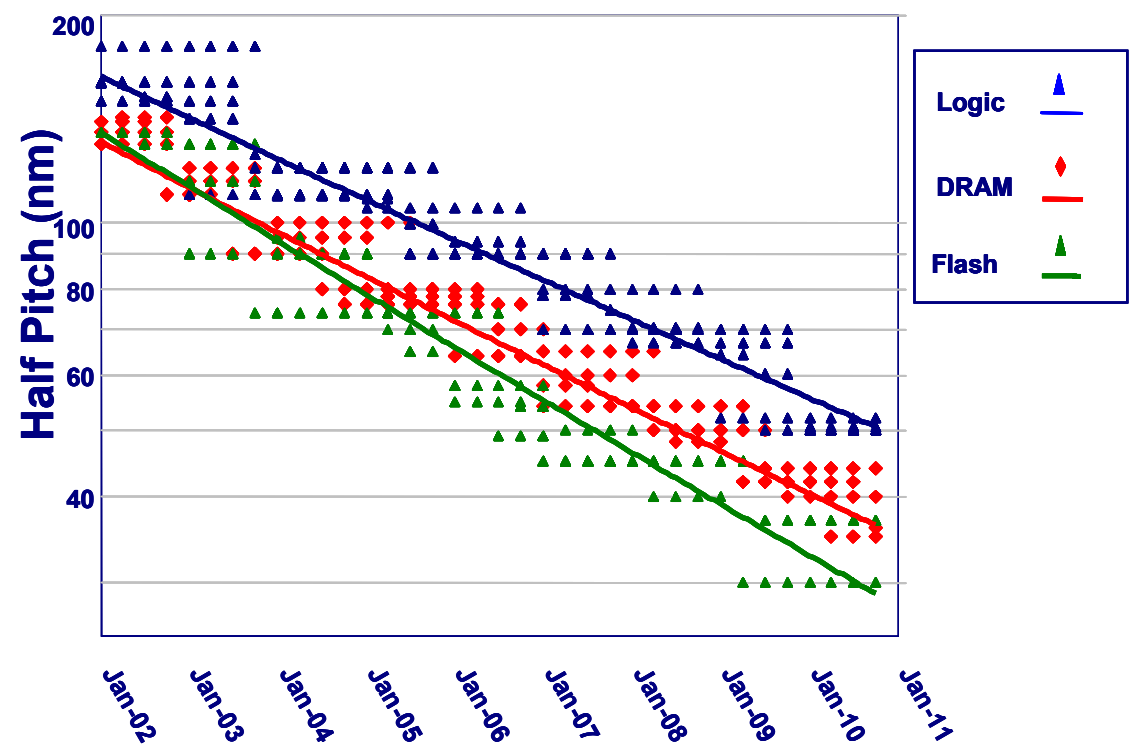

Fig. 1. Feature size reduction needed for development and manufacturing of ICs.

Current resolution improvements have been facilitated by the use of immersion technology to increase the NA limit beyond 1. If one assumes that the maximum angle in the fluid medium should not extend beyond $\sin \theta=0.95$ using $\lambda=193 \mathrm{~nm}$, then NAs up to $\sim 1.35$ can be contemplated when water is used as the immersion medium $\left(\mathrm{n}_{\text {water }}=1.437\right)$. However, if we also assume that higher index fluids $(\mathrm{n} \geq 1.6)$ may be available, then the maximum NA could easily approach 1.5.

The use of these NAs to extend imaging to sub-50nm levels will also require using polarization imaging to increase the overall contrast. This enables the use of single processing $\mathrm{k}_{1}$ 's approaching 0.25 when combined with a multitude of resolution enhancement techniques (RETs). One of these RETs that will likely be needed is source-mask optimization ${ }^{1}$. This technique simultaneously optimizes the shape of the source and the reticle features to effectively solve the inverse imaging problem. In addition, the use of double processing techniques effectively negates any lower limit is $\mathrm{k}_{1}$ factor. For example, a process that enables interleaving of gratings will half the k1 factor, such that the lower theoretical limit becomes $\mathrm{k}_{1}=0.125$. 
Figure 2 highlights the possible ranges of resolutions by use of equation (10) with $\lambda=193 \mathrm{~nm}$ and $\lambda=13.5 \mathrm{~nm}$ (EUV). For $\lambda=193 \mathrm{~nm}$, the use of immersion technology is clearly required to go to resolutions beyond $50 \mathrm{~nm}$. Extension much beyond $35 \mathrm{~nm}$ will require the use of multiple processing schemes to push the $\mathrm{k} 1<0.25$. The situation is different for EUV wavelengths. Here, the current system NA is 0.25 , but for even modest $\mathrm{k} 1=0.5$, the resolution approaches $30 \mathrm{~nm}$. Furthermore, the theoretical potential exists to go close to $10 \mathrm{~nm}$ using single processing techniques as the NA is increased to 0.35 . However, achieving this will involve substantial lens development and technology infrastructure.
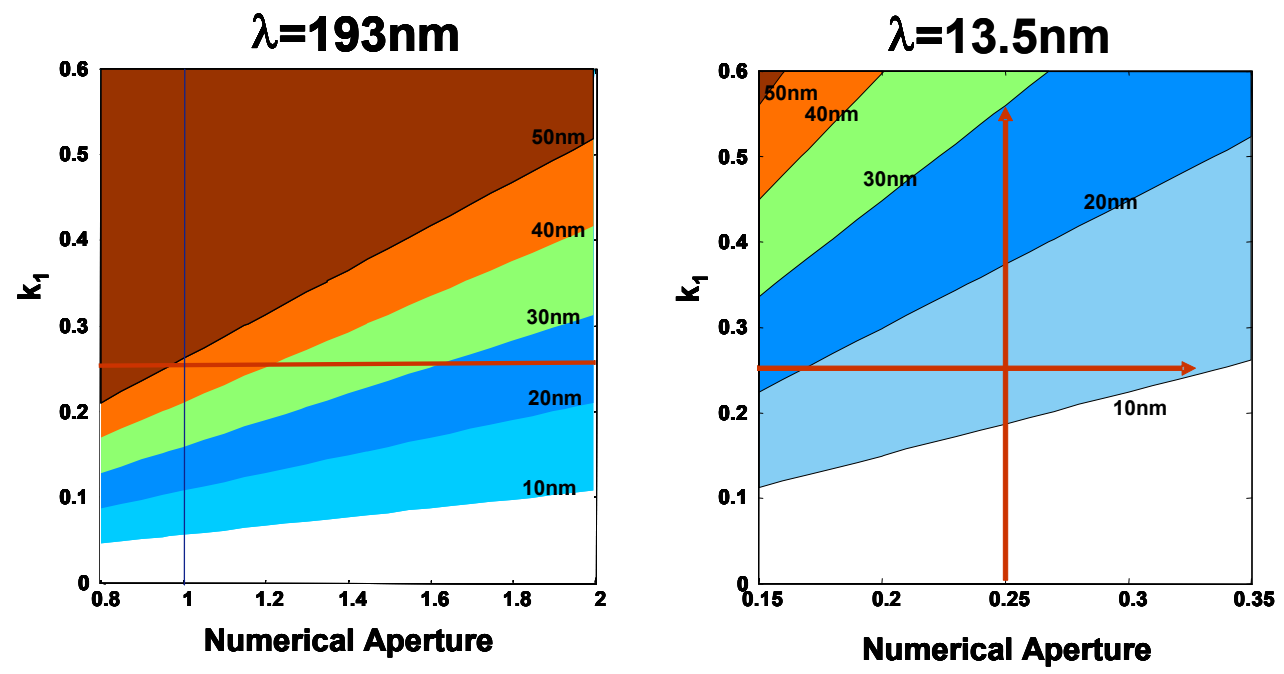

Fig. 2. Resolution space for $193 \mathrm{~nm}$ and EUV $(13.5 \mathrm{~nm})$ technologies

This paper will first show, in section 2, the general status of the state-of-the-art in immersion technology and the use of polarization imaging with hyper-NA lenses. Section 3 will explore a few of the methods used to lower $\mathrm{k}_{1}$ in single processing, while section 4 will discuss the current EUV system and results. Finally the last section shows recent advances in double processing techniques that will allow for resolutions well into the $10 \mathrm{~nm}$ regimes.

\section{IMMERSION LITHOGRAPHY AND HYPER-NA}

The use of immersion fluids in optics has been known for well over 150 years. In the late 1840 's, Giovanni Battista $\mathrm{Amici}^{2}$ filled the space between the microscope objective and the cover glass with oil to enhance the image quality and brightness. Since the oil matched the index of refraction of both the objective and the cover glass better than air, there was a reduction in spurious reflections that enhanced contrast. Amici also experimented with other fluids including water as the medium. However, it was not until 1880 that Ernst Abbe ${ }^{3}$ used a more carefully defined optical theory to specifically design microscopic objectives for immersion fluids.

Immersion for use in lithography first appeared about 100 years later in the patent by Taberrelli ${ }^{4}$ where a fluid that is indexed matched to the photoresist fills the area between the lens and the wafer. In 1987 Kawata $^{5}$ presented an optical projection system that used an oil immersion fluid at an exposing wavelength of $453 \mathrm{~nm}$. They were able to attain $160 \mathrm{~nm}$ isolated line features in Novalak photoresist using a research system based on an inverted microscope.

The use of interferometric systems for detailed exploration in lithography is well known ${ }^{6,7}$. Hoffnagle ${ }^{8}$ in 1999 extended this technique for immersion to increase the working resolution. He demonstrated line/space imaging in DUV photoresist. Subsequently, Switkes and Rothchild ${ }^{9}$ demonstrated interferometric immersion imaging at $157 \mathrm{~nm}$.

In more recent history, interference lithography coupled with immersion techniques has become a major method to achieve extremely high resolutions for periodic structures needed in some nanotechnology areas. This method is also extremely useful to explore various fluid materials needed for future generations of immersion tools. Figure 3 shows the results of an imaging test at ASML with a $2^{\text {nd }}$-generation fluid ( $1^{\text {st }}$ generation being water) using an immersion interferometer setup. Aggressive $32 \mathrm{~nm} \mathrm{L/S}$ imaging from this test setup is shown and some line edge roughness (LER) is 
observable, which is a property of the chemically amplified resists that is used. No adverse immersion fluid effects were observed in the resist profiles.

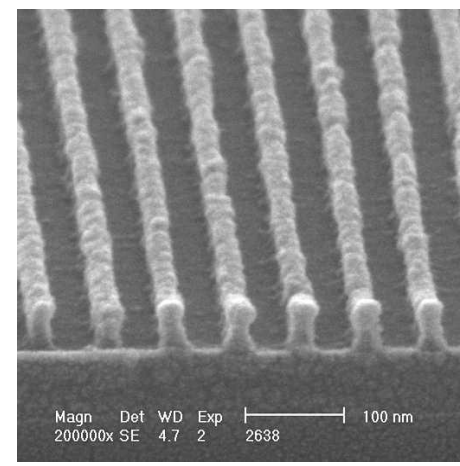

Fig. 3. Images of $32 \mathrm{~nm}$ line and space $(\mathrm{L} / \mathrm{S})$ patterns in resist using an immersion fluid with $n=1.65$. Two beam interference was used at $\lambda=193 \mathrm{~nm}$ and the sine of the half angle within the fluid was $\sin \theta=0.915$.

In order to explore the issues with water immersion lithography with a high volume full field system, in 2003 ASML and Zeiss modified the lens and wafer stage of an existing ArF $(\lambda=193 \mathrm{~nm})$ system (XT 1150, NA $=0.75)$ to produce the world's first scanned images using water immersion ${ }^{10}$. By introducing water between the last lens element and the wafer, we were able to demonstrate a substantial improvement in depth of focus over imaging in air. However, since the same lens design was used, there was no improvement in numerical aperture, hence no improvement in limiting resolution. In 2004 ASML introduced a second generation system, the XT 1250i (NA=0.85), and in 2005 we introduced the third generation system, the XT 1400i (ArF, NA=0.93). These immersion tools have been shipped to different customer locations around the world to facilitate early learning and process development.

The latest generation immersion system is the first hyper-NA system, i.e., NA $>1$. The TWINSCANTM XT:1700i, as depicted in Fig. 4 combines the immersion technology of the dual stage TWINSCAN ${ }^{\mathrm{TM}}$ platform ${ }^{11}$ with the new hyperNA projection system and new illumination system. The Starlith ${ }^{\mathrm{TM}} 1700 \mathrm{i}$ is the fourth generation of $193 \mathrm{~nm}$ immersion lenses and the first catadioptric lens from Carl Zeiss SMT. This lens operates in a NA range of 0.75 to 1.2 and supports a field size of $26 \times 33 \mathrm{~mm}^{2}$. The Aerial ${ }^{\mathrm{TM}} \mathrm{XP}$ illuminator supports a wide range of illumination settings, in different polarization modes and improved pupil parameters as ellipticity and telecentricity. For both polarized and un-polarized usage, the system has maximum efficiency for all supported illumination modes. The immersion hood is designed to support both hydrophobic and hydrophilic processing at $550 \mathrm{~mm} / \mathrm{s}$ scan speed. Table I gives the specifications of this tool.

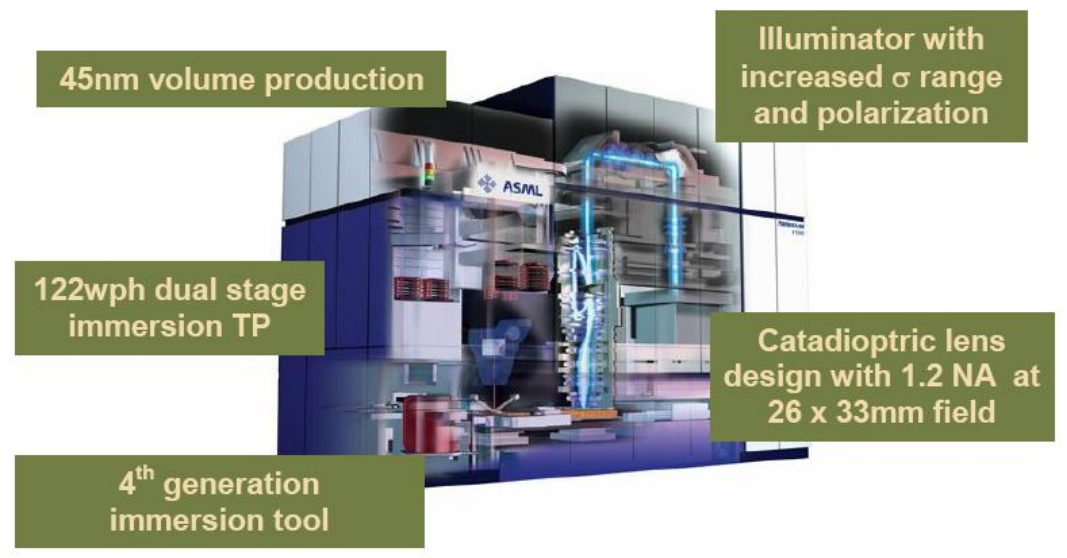

Fig. 4. ASML TWINSCAN XT:1700i $1.2 \mathrm{NA}$ with $26 \times 33 \mathrm{~mm} 2$ field 
Table I. Specifications of ASML Twinscan XT 1700i

\begin{tabular}{|c|c|c|c|}
\hline Lens & Spec & Illumination & Spec \\
\hline $\operatorname{Max} \mathrm{NA}$ & 1.2 & Laser & $6 \mathrm{kHz},>50 \mathrm{~W}$ \\
\hline Field Size & $26 \times 33 \mathrm{~mm}$ & Uniformity & $<0.3 \%$ \\
\hline Max RMS Aberration & $1.4 \mathrm{~nm}$ & & \\
\hline Astigmatism & $20 \mathrm{~nm}$ & \multicolumn{2}{|l|}{ Overlay } \\
\hline Focal Plane Deviation & $30 \mathrm{~nm}$ & Stage Repeatability & $4 \mathrm{~nm}$ \\
\hline Resolution, Annular & $50 \mathrm{~nm}$ & System Overlay & $7 \mathrm{~nm}$ \\
\hline Resolution, Dipole & $45 \mathrm{~nm}$ & Matched Overlay & $11 \mathrm{~nm}$ \\
\hline $\operatorname{CDU} 3 \sigma$ & $3.0 \mathrm{~nm}$ & & \\
\hline Distortion & $5 \mathrm{~nm}$ & \multicolumn{2}{|l|}{ Throughput } \\
\hline & & 125 shots, $30 \mathrm{~mJ} / \mathrm{cm} 2$ & $>122 \mathrm{WPH}$ \\
\hline
\end{tabular}

With 1.2 NA ArF optics, it is possible to manufacture 45nm design rule memories such as NAND flash devices with acceptable depth of focus and exposure latitude. Figure 5 shows 3 sets of images from this tool using 3 different illumination shapes $\left(1^{\text {st }}\right.$ column) to successively lower the $\mathrm{k}_{1}$ factor and increasing resolution.

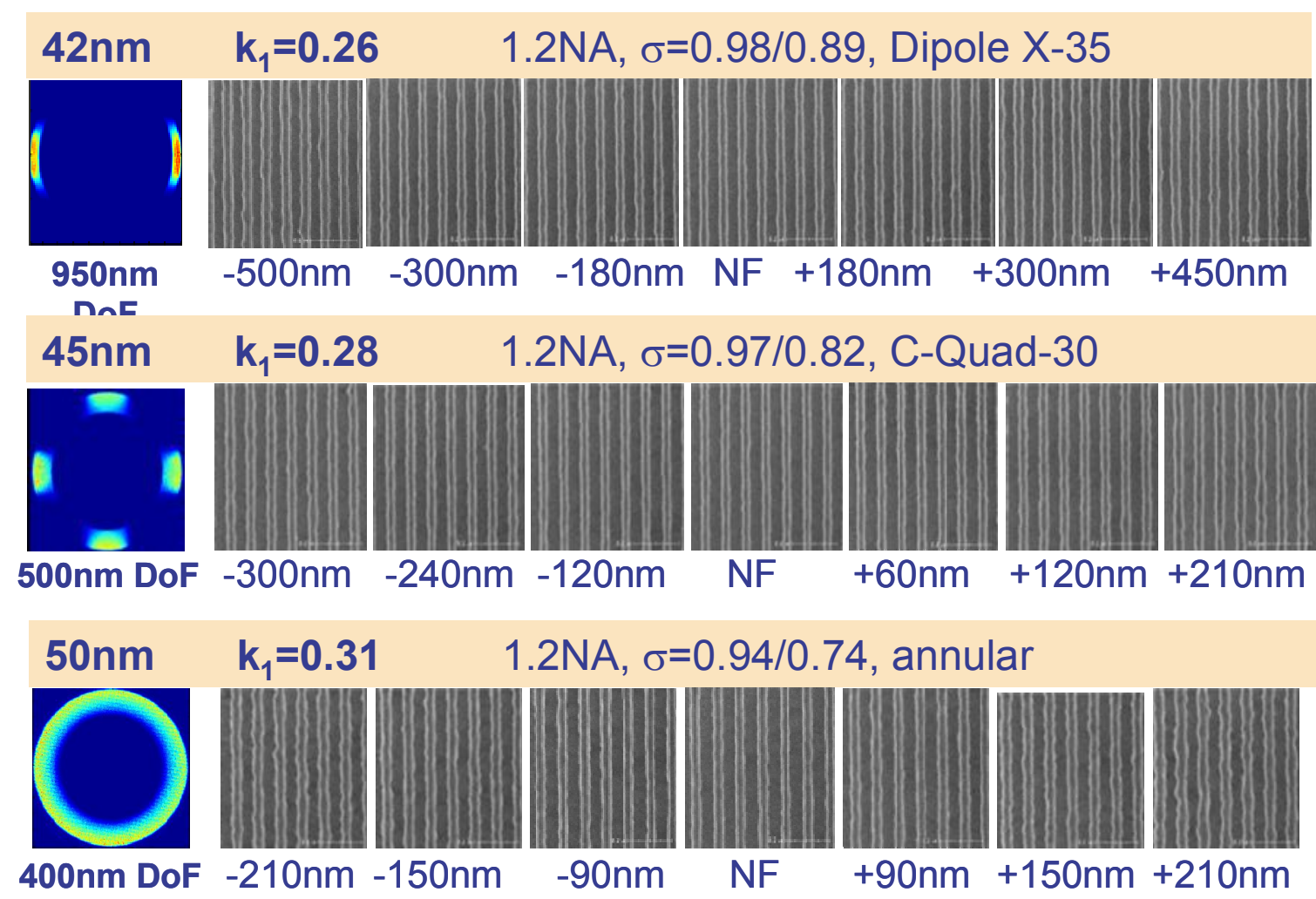

Fig. 5 Depth of Focus versus resolution for ArF lithography at NAs varying from 0.93 to 1.20

Development of hyper numerical aperture optics (NA $>1)$ has stressed the size and weight of optics. To date, most scanner optics from ASML and it's competitors have been dioptric designs, in which only refractive elements of either fused silica or calcium fluoride have been used. In order to control the complexity and cost of the projection optics, Zeiss has designed the 1.2 NA lens to be an in-line catadioptric lens, using both mirrors and refractive elements. A crosssectional view of an example of this design is shown in Fig.6. This design has allowed a large aperture to be built within the maximum length and diameter of previous lenses. ASML and Zeiss chose the in-line catadioptric design over further 
extension of dioptric form because it offered $15 \%$ more NA while saving $40 \%$ in glass material. Compared to other catadioptric forms, the in-line design allows easy extendibility to NA $>1.35$, offers a rectangular scan field which supports maximum productivity, retains the same image orientation as refractive lenses, and has high mechanical stability.

Imaging at very high NA values will require that polarization effects need to be taken into account as well. This is graphically illustrated in figure 7 for 3 polarization conditions: transverse electric (TE), transverse magnetic (TM), and the unpolarized condition. The contrast is calculated inside a photoresist of index $\mathrm{n}_{\text {resist }}=1.7$ as function of NA using a 2-beam interference assumption. This approximates imaging dense lines with an alternating phase mask using a small partial coherent illumination. Although the TE case will have maximum interference resulting in a contrast of $100 \%$, the TM case will have a contrast that decreases by the cosine of the angle between the beams. The contrast within the resist will drop to 0 at $\mathrm{NA}=1.2$ and increasing the NA further will result in image reversal for TM. Since the unpolarized condition is the average of the TE and TM polarization, the unpolarized case will also suffer from an image degradation that increases with NA.

The illumination system of the ASML TWINSCANTM XT:1700i supports polarized illumination modes in order to get to full benefit of the 1.2NA. The AERIAL $^{\mathrm{TM}}$ XP illuminator of system supports both polarized and un-polarized illumination with the same efficiency. All future systems at these extreme NAs will require some amount of polarizability to restore lost contrast. Figure 8 shows polarized illumination achieving an improvement of 30\% exposure latitude (EL) and almost 60\% improvement in maximum DOF.

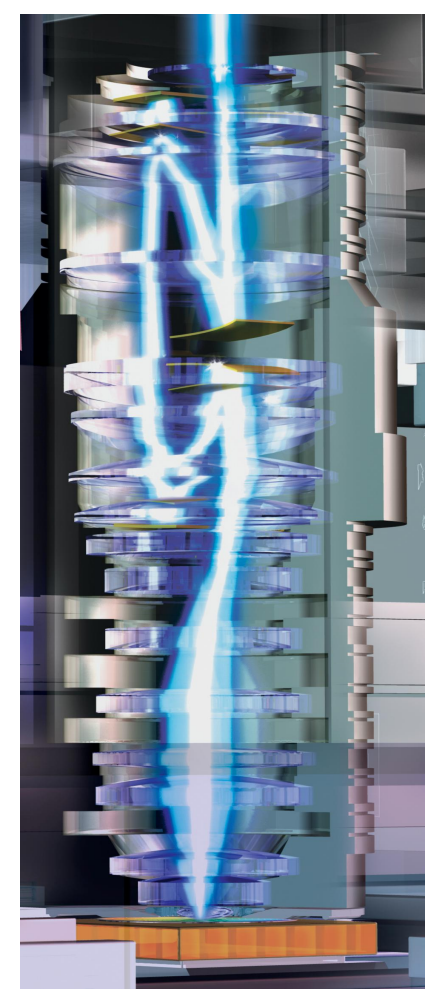

Fig. 6. Starlith ${ }^{\mathrm{TM}} 1700 \mathrm{i}$ In-Line Catadioptric Lens, maximum $\mathrm{NA}=1.2$.

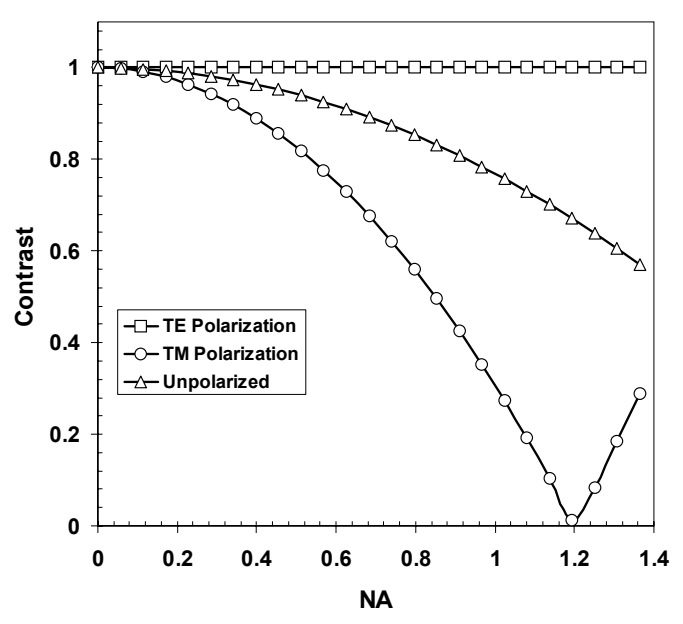

Figure 7. Contrast of 2 beams interfering within photoresist film of index 1.7. The half angle between the beams is given as the NA.

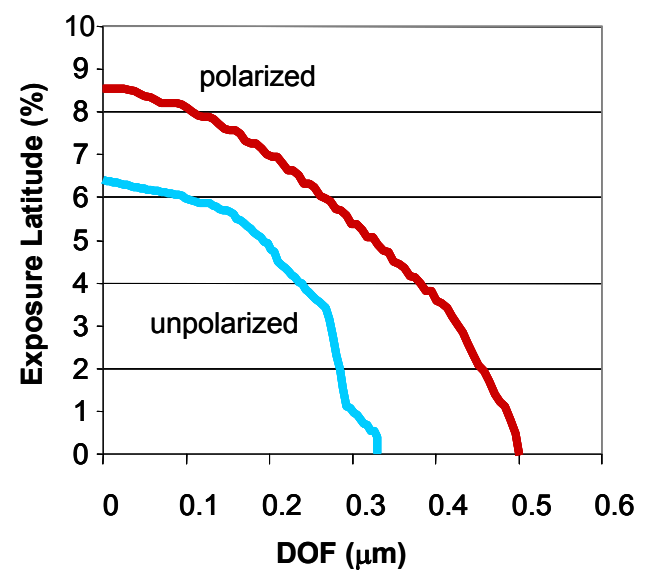

Fig. 8. 45nm dense $\mathrm{H} \& \mathrm{~V}$ lines using TE polarized CQuad illumination 1.2NA, s=0.94/0.87 . 


\section{LOWERING $k_{1}$ BY RESOLUTION ENHANCEMENT TECHNIQUES}

As a consequence of pushing the technology to lower and lower resolutions using lower $\mathrm{k}_{1}$ values, the image contrast degrades, as well as, the size and shape of the final image. Short of going to the next NA or wavelength (equivalent to increasing $\mathrm{k}_{1}$ ), one has two main choices how to improve the image: change the shape and size of features on the mask and / or optimize the illumination. The first choice is known as optical proximity correction (OPC) in which the edges of mask features are purposely moved with respect to the CAD layout in order to achieve a better final wafer result.

One of the more recent approaches for periodic structure involves optimization of the illumination shape. For memory devices, pattern density is paramount, putting significant restrictions on available options for OPC. For these cases, illumination optimization is very attractive. Figure 9 shows how the lithography process window for a typical DRAM isolation layer can be improved by using an optimized illuminator customized for that layer compared to more standard illumination types such as annular.

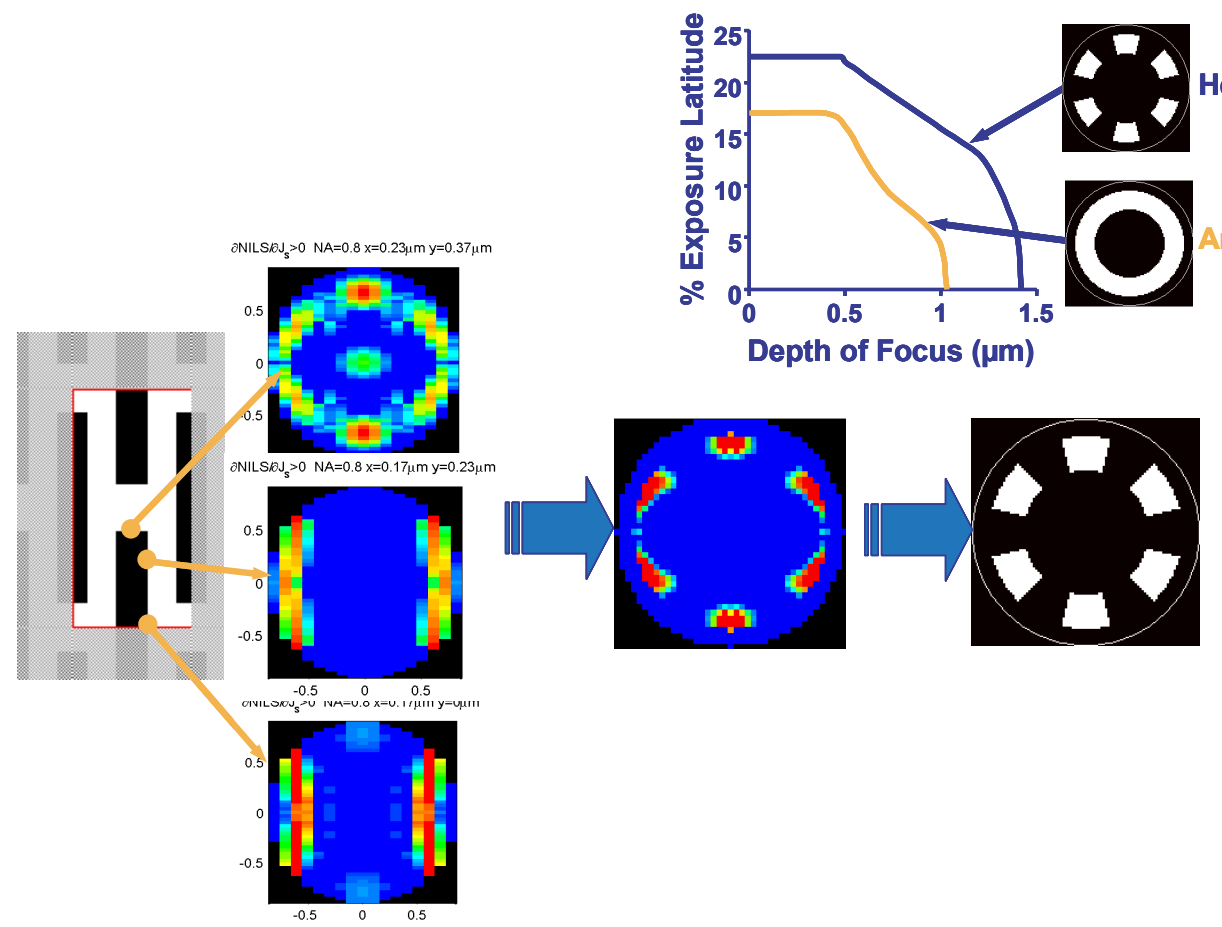

Fig. 9. The illuminator shape is designed by finding the optimum illuminator for each critical edge point, then doing a weighted sum to come up with a total illuminator shape. In this example a hexapole shape was optimum as compared to an annular shape.

At a further level of sophistication, it is possible to vary both mask layout and optimize illumination simultaneously, i.e., source-mask optimization $^{1}$ (SMO). This technique is based on solving the inverse imaging equation by simultaneously deriving an optimized illumination and reticle for a given optical system. An example of this approach for a sub-50nm brick-wall feature using annular illumination is shown in figure 10a. This feature has a critical line width at $45 \mathrm{~nm}$ with a $90 \mathrm{~nm}$ pitch. The line length is $288 \mathrm{~nm}$ with an end gap of $72 \mathrm{~nm}$. In best focus, at the nominal exposure, the annular illumination shows bulging line ends with a gap of $91.6 \mathrm{~nm}$ or a $27.2 \%$ increase of the desired gap. Using an internal ASML algorithm, the optimum mask and illumination design can be calculated and are shown in figure 16b. The shape of the brick wall with the optimization routine is much closer to the desired shape. The gap now is $82.2 \mathrm{~nm}$ or an increase in the gap of $14.2 \%$. This is approximately a $2 \mathrm{x}$ improvement in the end-of-line. 


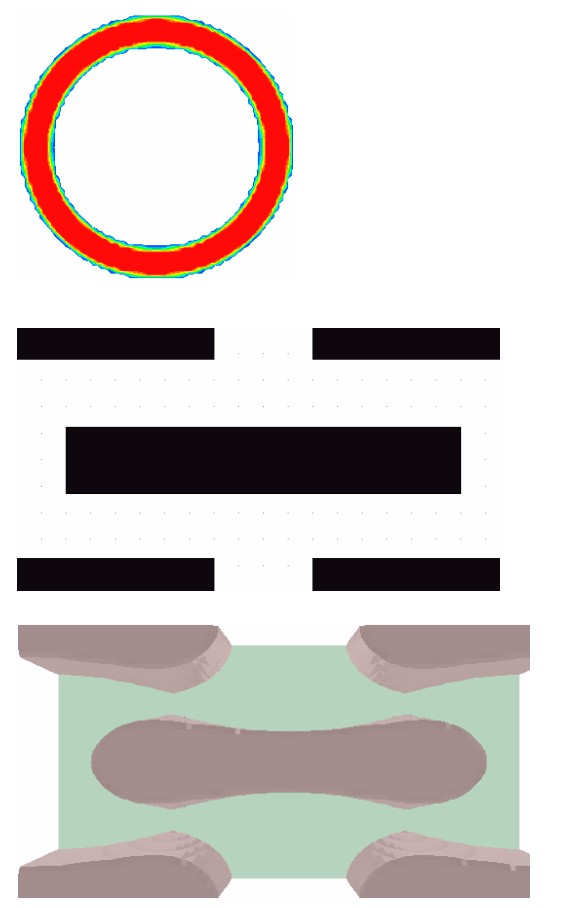

Fig. 10a. (Top) annular illumination source with souter $=0.95$ and sinner $=0.75$; (middle) traditional brick wall pattern; (bottom) resultant topdown photoresist pattern.
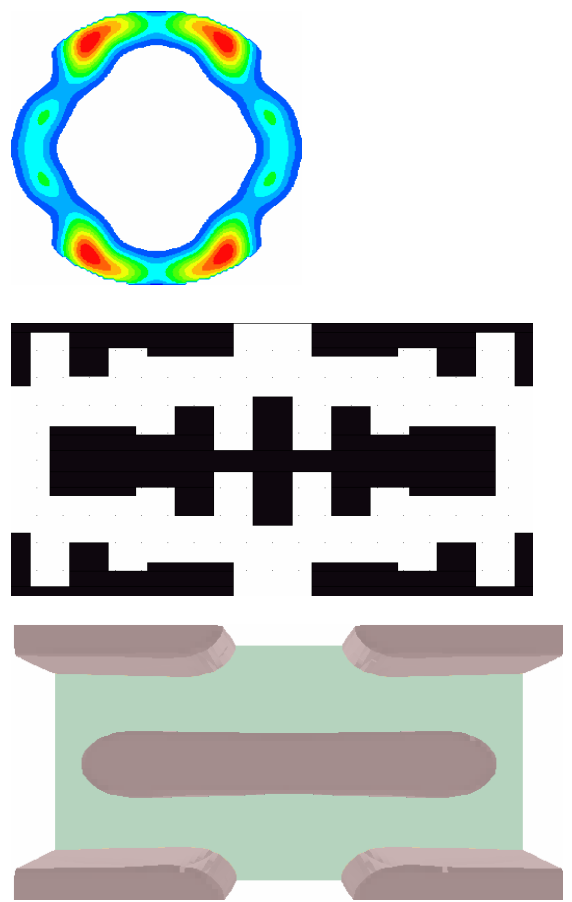

Fig. 10b. (Top) optimized illumination source; (middle) optimized brick wall pattern; (bottom) resultant top-down photoresist pattern.

In logic devices, as in many practical cases, the device is not laid out on the densest pitch possible and the critical features are often relatively isolated from neighboring features in an optical sense. The process window for printing small isolated features is usually worse than that for dense features due to small depth of focus. A very successful approach to improving process window for logic structures as might be found at, for example, gate level of a system on chip (SoC) device is to add scatter bars ${ }^{12}$ to the mask. Scatter bars are sub-resolution mask features which improve the printing fidelity of isolated features by making them "look dense". Combined with off-axis illumination, scatter bars can increase the size of a lithography process window substantially. Automated software is available to place and size scatter bars on a given CAD layout, as well as to recommend optimized NA and illumination parameters for scanner settings (ASML Masktools product LithoCruiser ${ }^{\mathrm{TM}}$ ).

\section{EUV TECHNOLOGY}

Tremendous progress has been realized in the past year in all key areas of EUVL development. EUV systems operate at $\lambda=13.5 \mathrm{~nm}$ and must be in vacuum. The reticle technology, as well as the lens and illuminator technologies, uses all reflective components due to the lack of any exiting materials with acceptable transmission at this wavelength. As previously seen in figure 2, the potential gain in resolution with EUV is large, but, the realization of large scale use of this technology is not trivial. However, several small-field exposure tools are now operational around the world, and ASML has built the world's first 0.25 NA large field EUV system that is capable of use in a high volume production facility. This is an alpha demo (AD) tool and is now in the final phase of imaging qualification. The main objectives for building this tool are to provide a means to utilize this new lithographic wavelength such that risks associated with changing to a new technology can be minimized. Thus, this tool is designed to support the global development of needed infrastructure of reticles, illumination sources, and photoresists. 
The alpha demo tool is fully assembled and shown in figure 11. All modules required for lithography have been integrated, and the system's metrology sensors, wafer- and reticle stages, and material handling modules have been qualified. The complete optical train comprising the illuminator and projection optics is integrated and is currently being qualified.

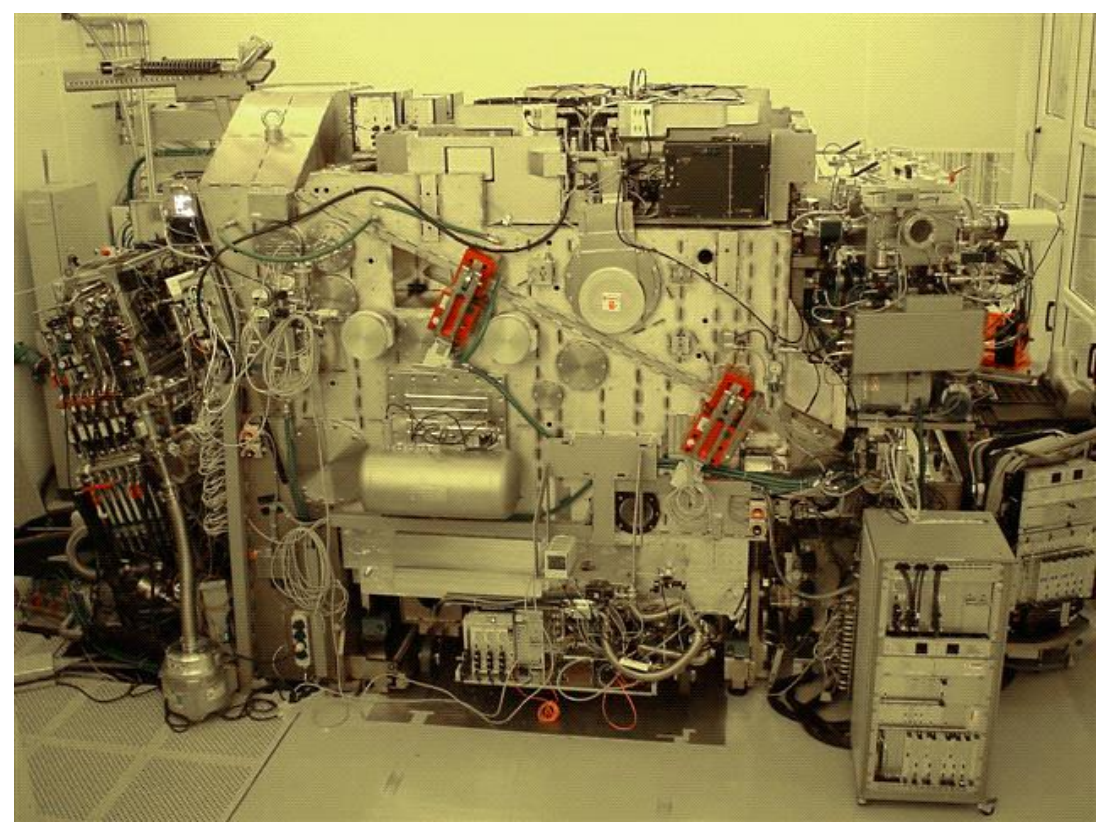

\begin{tabular}{|l|l|}
\hline & AD-tool \\
\hline$\lambda$ & $13.5 \mathrm{~nm}$ \\
\hline NA range & $0.15-0.25$ \\
\hline Field size & $26 \times 33 \mathrm{~mm}^{2}$ \\
\hline Wafer size & $300 \mathrm{~mm}$ \\
\hline Magnification & $4 \times$ \\
\hline Flare & $16 \%$ \\
\hline Dense L/S & $40 \mathrm{~nm}$ \\
\hline Isolated lines & $30 \mathrm{~nm}$ \\
\hline Iso/dense contact & $55 \mathrm{~nm}$ \\
\hline Overlay & $12 \mathrm{~nm}$ \\
\hline Throughput & $\sim 10 \mathrm{wph}$ \\
\hline
\end{tabular}

Figure 11. Picture of the ASML EUV alpha demo tool and a summary of characteristic tool parameters. All modules needed for lithography have been integrated, and testing to system specifications is in process.

The ASML EUV alpha demo tool builds on TWINSCAN ${ }^{\mathrm{TM}}$ technology, adding in new modules and capabilities that are unique to EUVL. In some cases, sensors, such as used for alignment and registration, must be made vacuum compatible. In other areas, like reticle handling, an entire new module design has been developed to address particle-free transfer of reticles from atmosphere to vacuum. Key architectures such as software, electrical, and temperature control are based on TWINSCAN ${ }^{\mathrm{TM}}$, but modified to address the additional components for EUV. The vacuum system architecture is completely new. By building on the previous architecture and modules, we have been able to focus on the development of EUV-unique areas, while taking advantage of hardware that has been proven on existing ASML products. The ADtool has been targeted for early learning of EUVL, hence it is a low throughput tool with a single stage. Still, both the reticle stage and wafer stage design concepts are extensible for high throughput EUVL.

Initial imaging results are presented in figure 12. These are $40 \mathrm{~nm}$ periodic lines demonstrating more than $20 \mathrm{~nm}$ DOF., through a focus series. Figure 13 shows these lines at best focus, but through the extent of the image field. Finally, figure 14 shows perhaps one of the greatest strengths of EUV, the ability to print dense and isolated contacts, simultaneously at the same exposure dose and focus resulting in the same dimension without the need for OPC. This is a direct consequence of imaging at high $\mathrm{k}_{1}$.

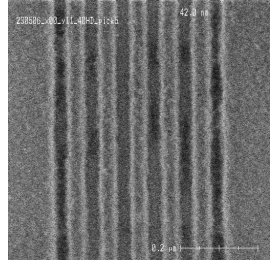

$-100 \mathrm{~nm}$

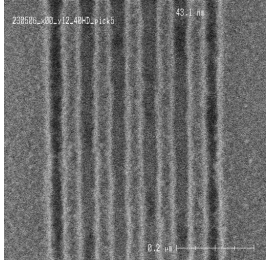

$-50 \mathrm{~nm}$

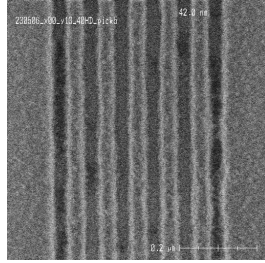

near focus

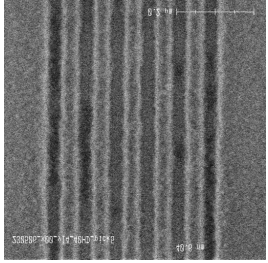

$+50 \mathrm{~nm}$

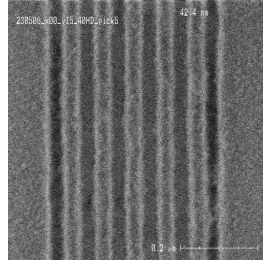

$+100 \mathrm{~nm}$

Fig 12. SEM pictures showing $>200 \mathrm{~nm}$ depth-of-focus for $40 \mathrm{~nm}$ lines. (MET-2D resist, NA=0.25; $\sigma=0.5$ conventional illumination). 


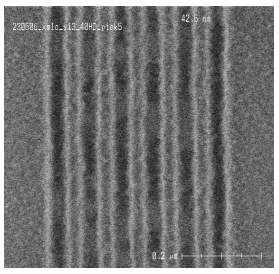

$-10.6 \mathrm{~mm}$

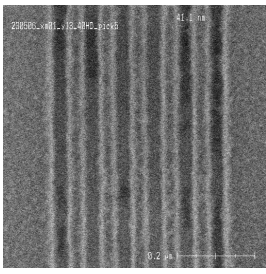

$-6.36 \mathrm{~mm}$

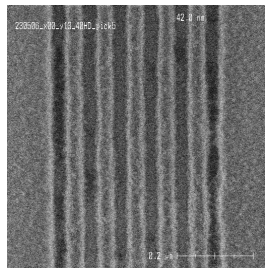

$0.0 \mathrm{~mm}$

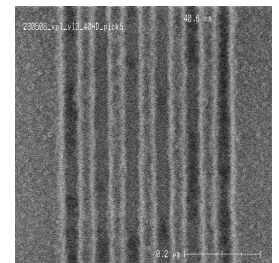

$6.36 \mathrm{~mm}$

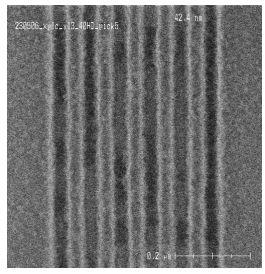

$10.6 \mathrm{~mm}$

Fig. 13. SEM pictures showing full field coverage for $40 \mathrm{~nm}$ lines. (MET-2D resist, $\mathrm{NA}=0.25 ; \sigma=0.5$ conventional illumination).

Dense (aligned)

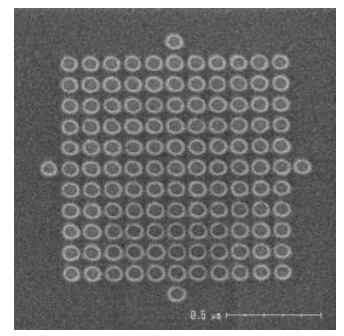

Dense (staggered)

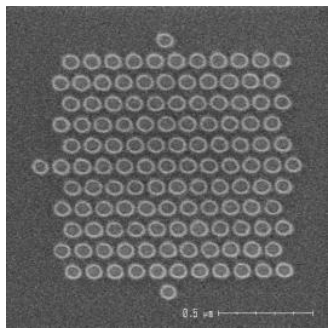

Iso (aligned)

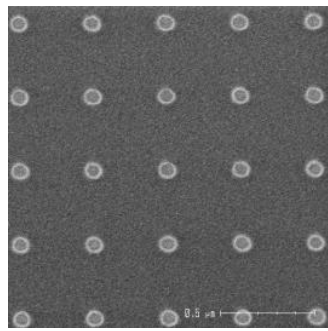

Iso (staggered)

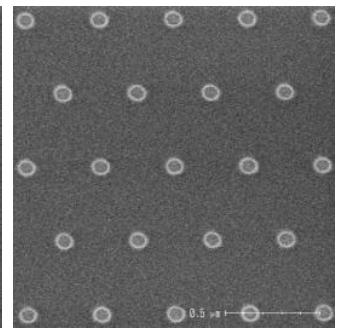

Fig. 14. 55nm dense and isolated contact holes print simultaneously - same dose \& focus.

\section{THE PROMISE OF DOUBLE PROCESSING TECHNOLOGY}

Over the next few years, $193 \mathrm{~nm}$ lithography buttressed by the twin pillars of hyper NA immersion optics and low $\mathrm{k}_{1}$ technology, will continue the reduction shrink required by leading volume producers of integrated circuits. In 2007, ASML will introduce a tool with $\mathrm{NA}=1.35$ which will allow mass production of memory devices at $40 \mathrm{~nm}$ design rule. However, the refractive index (n) of water establishes the upper limit of NA which can be achieved in water immersion lithography at approximately 1.4. An aperture of at least 1.6 is required to image $32 \mathrm{~nm}$ half-pitch resolution with $\mathrm{k}_{1} \sim$ 0.28 , the current benchmark for mass production with good yields. Higher apertures will require the introduction of new fluids and possibly new glasses with higher refractive index.

Some progress has been made in identifying a class of fluids with $\mathrm{n} \sim 1.64$. However, an immersion fluid also has to satisfy a number of other physical and economic requirements as well. For example, it must have low absorption, it must have low $\mathrm{dn} / \mathrm{dT}$ (small change of $\mathrm{n}$ with temperature), it must have viscosity and wetting characteristics suitable for high speed scanning, and it must be readily available for relatively low cost. When one considers the set of stringent requirements which must be met, one is struck with what a miracle fluid is water!

Likewise, it is difficult to find new high index glasses which could replace fused silica for the bottom lens element. Current focus is on materials such as garnets (e.g., LuAG) which have $n>2$, but have very high intrinsic birefringence (IBR), a material property that makes light polarized along one axis travel slower through the material than light polarized along an orthogonal axis. Such high IBR leads to unacceptable imaging degradation. It is not currently clear if the lens designers can find ways to compensate for these effects. 
If no new fluids or glasses are found and NA is limited to $\sim 1.4$, then what are some other ideas which may allow further extension of $193 \mathrm{~nm}$ lithography? One idea which has attracted attention in the last couple of years is the use of double exposure (DE) and double patterning technologies (DPTs). Although the use of two exposures can enable a smaller $\mathrm{k}_{1}$ to be achieved, it is not possible to resolve features at $\mathrm{k}_{1}$ less than 0.25 even if two exposures are used in a single resist step since the combined image has zero contrast.

The DPTs that go beyond the $\mathrm{k}_{1}=0.25$ barrier usually rely on splitting the frequencies of the pattern, and then treating each split frequency as separate lithographic images. Intermediate steps that contain developed photoresist are etched to transfer the $1^{\text {st }}$ intermediate image. The result is an interleaving of the patterns that doubles the pitch. An example of this process and experimental results indicating resolution of grating patterns down to $\mathrm{k}_{1}=0.19$ have been demonstrated at ASML (shown in figure 15), IMEC ${ }^{13}$, and by Ebihara ${ }^{14}$. Alternatively, each exposure can be done with 1 photoresist develop step, but this requires a fixation step to stabilize and threshold the $1^{\text {st }}$ image such that it does not interact with the $2^{\text {nd }}$ exposure.
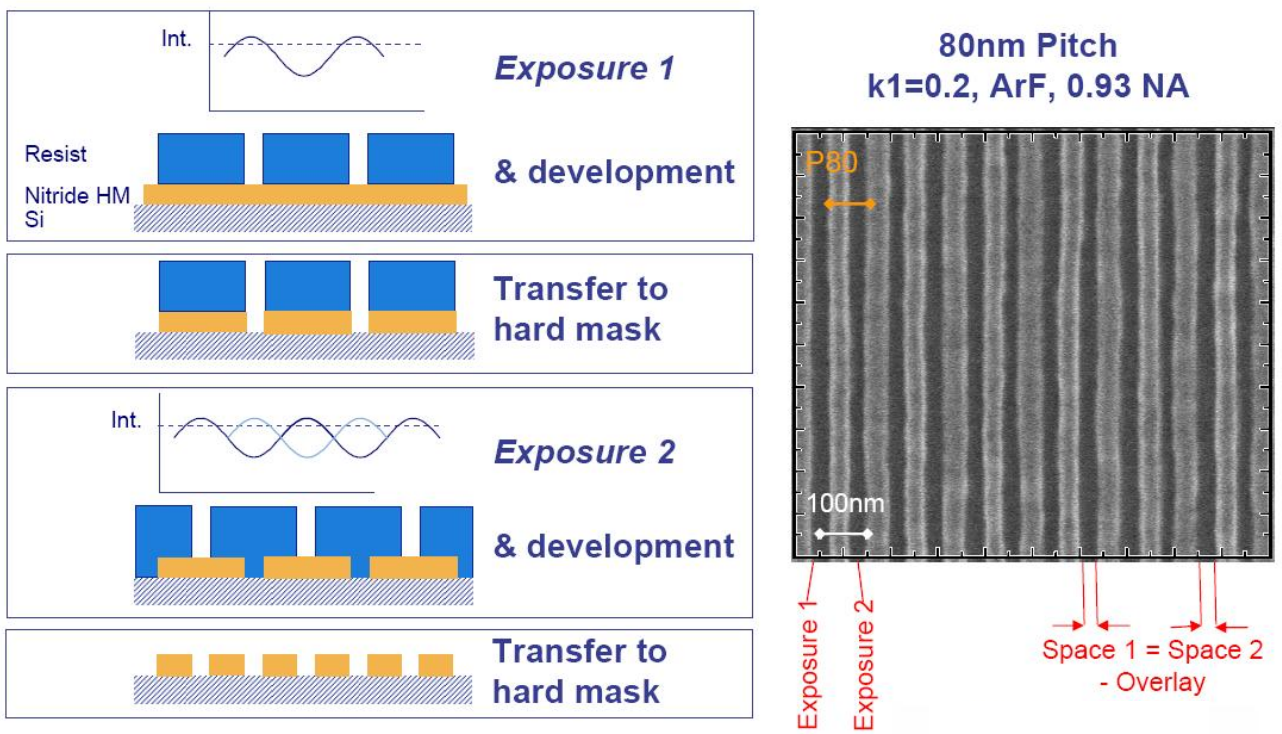

Fig. 15. Principle of Double Patterning: a method to break the effective $0.25 \mathrm{k}_{1}$ barrier

The advantage of DPT is that it is a relatively straightforward extension of existing technology - no new fluids, glasses, reticles, exposure sources, resists, etc. are needed to make it work. However, there are several disadvantages, a few we list here: two (or more) reticles are needed for each critical layer, and twice the number of exposures must be done effectively cutting scanner productivity by half. Thus it is clear that cost of ownership of advanced lithography will be challenged. In addition, double patterning brings with it very tight overlay requirements as overlay errors are now directly part of the critical dimension budget. A further critical issue is the availability of sophisticated design splitting software which can deconstruct a complicated pattern into two complementary patterns which can be recombined through double imaging without errors.

The technology is not limited to $193 \mathrm{~nm}$ wavelengths. Indeed, one can envision the use DPT to proceed down the resolution evolutionary ladder towards sub- $10 \mathrm{~nm}$ using a future EUV lens with $\mathrm{NA}=0.35$ and $\mathrm{k}_{1}=0.5$ ! 


\section{CONCLUSION}

Optical lithography continues to provide means and methods to produce nanoscale devices on a grand scale. Recent developments in immersion, hyper-NA, EUV, and multiple patterning lithography continue to open new avenues for development and manufacturing. There are considerable challenges for further extension of 193nm lithography in both the development of high index materials to drive apertures beyond 1.4, and in further extension of low $\mathrm{k}_{1}$ technology through double exposure (DE) / double patterning (DPT). Momentum of recent developments makes it likely these further extensions will be attempted - at what point we have to give up and say we need to move to the next wavelength (13.5 $\mathrm{nm}$ or EUV), will be the next great debate in the lithography community.

\section{REFERENCES}

1. A. Rosenbluth et. al., "Optimum mask and source patterns to print a given shape", Proc. SPIE, vol.4346, pp.486-502 (2001)

2. S. Bradbury, Evolution of the Microscope, Pergamon Press, Oxford (1967)

3. R. Kingslake, Optical System Design, pp. 188-190, Academic Press, Orlando (1983)

4. W. Taberelli and E. Loebach, US Patent 4,346,164, issued Aug 4, 1982

5. H. Kawata, J. Carter, A. Yen. H. I. Smith, "Optical Projection Lithography using Lenses with Numerical Apertures Greater than Unity", Microelectronic Engineering 9 (1989), pp. 31-36

6. S. H. Zaidi and S. R. J. Brueck, "Multiple exposure interferometric lithography", Proc. SPIE, vol. 2197, pp. 869-875 (1994)

7. D. G. Flagello and T. D. Milster, “ High-numerical aperture effects in photoresist”, Applied Optics, vol. 36, No.34 (1997), pp 8944-8951

8. J.A. Hoffnagle, W.D. Hinsberg, M. Sanchez and F.A. Houle, "Liquid Immersion deep-ultraviolet interferometric lithography", J.Vac.Sci.Techn. B 17(6), Nov 1999

9. M. Switkes and M. Rothschild, "Immersion Lithography at 157nm", J.Vac.Sci.Techn. B 19(6), Nov 2001

10. B. Streefkerk et. al., "Extending optical lithography with immersion", Proc. SPIE, vol. 5377, pp. 285-305, 2004

11. B. Sluijk, "Performance results of a new generation of 300mm lithography systems", Proc. SPIE, vol. 4346,1999

12. J. Fung Chen,US Patent 5,242,770

13. M. Maenhoudt et. al., "Double patterning scheme for sub- $0.25 \mathrm{k}_{1}$ single damascene structures at NA=0.75, $\lambda=193 \mathrm{~nm}$ ", Proc. SPIE, vol. 5754, 2005

14. T. Ebihara, M.D. Levenson et al, Proc. SPIE, Vol. 5626, 985-994, 2003 